\title{
GeRd SPITTLER
}

\section{Herrschaft über Bauern}

Die Ausbreitung staatlicher Herrschaft und einer islamisch-urbanen Kultur in Gobir (Niger) Campus Verlag (Campus Forschung Band 49), Frankfurt/New York 1978, 190 S.

Spittler beschreibt in seinem Buch, das ein Ergebnis von drei längeren Aufenthalten des Autors in einer von Hausa sprechenden Bauern bewohnten Region Nigers darstellt, einmal die Ablösung einer autonomen Agrarkultur durch eine städtisch-islamisch geprägte Kultur. Er stellt diesen Prozeß in den Zusammenhang der Expansion des Islams und der Zunahme der agrarischen Exportproduktion, verfolgt die Auswirkungen bis in kleinste Verästelungen des täglichen Lebens und konstatiert am Ende in vielen Bereichen des sozialen und ökonomischen Lebens ein wachsendes Stadt-Land-Gefälle.

Hochinteressant ist die Analyse des Verhältnisses zwischen Bauern und Verwaltung, die den Hauptteil der Arbeit ausmacht. Nach einem knappen Überblick über die jüngste Geschichte Nigers und die historische Entwicklung sowie die sozio-politischen Strukturen der untersuchten Region stellt Spittler die Perzeption von Herrschaft aus der Perspektive der Bauern dar und analysiert deren Reaktionen auf Handlungen staatlicher Instanzen. Hier kann er eine Fülle neuer Erkenntnisse vorlegen, die nur aufgrund eines langen und intensiven gemeinsamen Lebens mit den Bauern gewonnen werden konnten ( $\mathrm{da}$ ß für Spittler bei seinen Forschungen vor Ort ,,Teilnahme“ gleichrangig mit ,,Beobachtung"war, wird bis in die Beschreibung der physischen Belastung der Bauern bei der Feldarbeit spürbar).

Selten ist die ,Sprachbarriere" $z$ wischen den zentralen staatlichen Instanzen und den Bauern in einem afrikanischen Land in ihren Ursachen und verheerenden wirtschaftlichen und politischen Folgen so einsichtig herausgearbeitet worden. Die Bauern interpretieren Herrschaft als quasi naturgegebenes Gewaltverhältnis, in dem die Herrschenden eindeutig überlegen sind und aufgrund ihrer Machtposition ihre Handlungen nicht weiter legitimieren brauchen (z. B.durch Leistung oder dadurch, daß ihre Anordnungen einsichtig sind). Da die Bauern aufgrund ihres Herrschaftsmodells - Spittler charakterisiert es als ,, asymmetrisches Nullsummenspiel“" - den Staat bzw. die Verwaltung primär als Steuereintreiber wahrnehmen, von dem keine Gegenleistung zu erwarten ist, versuchen sie durch verschiedene Mittel wie Ignorieren oder Deformieren von Anordnungen oder scheinbarer Zustimmung zu ihnen die unterstellten Nachteile zu unterlaufen bzw. der Verwaltung soweit als möglich auszuweichen. Sehr plausibel wird dargelegt, daß allein aufgrund unterschiedlicher Erwartungshaltungen von Bauern und Zentralverwaltung Entwicklungsprojekte bereits im Ansatz zum Scheitern verurteilt sind. Die Verwaltung vor Ort versucht erst gar nicht, Termini wie ,, Partizipation“ und ,Dialog" begreifbar zu machen, sondern beschränkt sich darauf, durch die Demonstration ihrer Macht die Bauern zur Durchführung bestimmter Projekte zu veranlassen, während diese nach dem üblichen Muster den Maßnahmen der Verwaltung auszuweichen versuchen.

Das Buch wird abgerundet durch einen Anhang mit der Darstellung ausgewählter ökonomischer Fragen, die die Bauern betreffen (ihr Budget, die Kommerzialisierung des Bodens etc.) sowie mit Preisliedern, die einen weiteren Einblick in die politische Kultur der Hausa-Bauern geben.

Klaus Ziemer 